\title{
Correction of Deformed Images based on Deformation-Map
}

\author{
Dhafer R. Zaghar, PhD \\ Assist. Prof., University of Al- \\ Mustansiriyah / Collage of \\ Engineering \\ Iraq/Baghdad
}

\author{
Mohammed Ali Tawfeeq \\ Collage of Engineering/ \\ University of Al-Mustansiriyah \\ Baghdad - Iraq
}

\author{
Ihab Ibrahim Kadhim \\ University of Al- Mustansiriyah / \\ Collage of Engineering \\ Iraq/Diyala
}

\begin{abstract}
In the last years the development in digital image acquisition, capturing, processing and displaying devices is one of the fastest growing fields. Most of these devices introduce some amount of geometric lens deformation and cause uncorrected dimensions in displaying the objects, such as cameras, scanners, lenses, and mirrors. This type of deformation is main issue in computer vision and in many image analysis systems, thus the availability of accurate image positional information is requisite. This work introduces a general algorithm use to correct the images deformed by a fixed and known properties system. A simple prototype system (convex mirror with digital camera) has been constructed, in which the mirror and the camera are well positioned to each other to capture any required reflected scene or image. The idea of the proposed algorithm is based on selecting an original image, capturing its reflection, and then obtaining the deformed ratios for each row and column between the captured image and the original image. These ratios represent the raw components to what referred here as the deformation-map. This map is assumed to be a general pattern for the proposed prototype system, to be used later in correcting the deformed images. The correction process depends mainly on three operations; stretching-padding-cropping. In the first operation, each row and column is stretched to a length depends on the corresponding compression ratio's element in the deformation-map. The second operation modifies the size of the image by adding pad array to the end sides, while the third operation crops the image to the required size. The values of the expanded pixels are computed using some interpolation method. Very satisfactory correction results have been obtained using the extracted deformation-map. Moreover, it is found that this map can be applied to correct deformed grayscale, RGB, and binary images.
\end{abstract}

\section{General Terms}

Deformed images, Image correction algorithm.

\section{Keywords}

Convex mirror, Deformed image, Corrected image, Deformation-Map.

\section{INTRODUCTION}

Optical lens and convex mirror systems generally contain non-linear deformation that imposes important limitations on the interpretation of the images. These deformations are often undesirable for a variety of applications in image processing and computer vision, such as image alignment, image merging, human identification, and accurate 3-D measurement, so it is necessary to correct the position of the pixels and correct the dimensions.
The deformation in digital image is changing the position and value of image element (pixels), in other words, it is moving image points locally and causes error in positions and values of image points. The lens deformation may be classified in to two types, radial deformation and tangential deformation. A radial deformation causes image points to be displaced from their ideal position toward (barrel deformation) or away from the image center (pincushion deformation), while the tangential deformation causes image points to be displaced in the direction perpendicular to the line connecting them to the image center. The radial distortion is caused by the imperfect lens design or by using mirror [1].

In general, when talking about lens deformation it usually meant the radial deformation that causes the image to look bowled out (barrel deformation) or bowled in (pincushion deformation).To compensate for these effects algorithms usually use low-order polynomials to re compute the positions for each pixel based on its distance to the image. This and more complex lens correction algorithm where introduces in several literatures. Yusoff et al, [2] proposed a conic fitting method for establishing distorted straight-lines of different orientation including the critical vertical and horizontal lines without having to introduce piecewise scheme, this process can accommodate various line orientations from vertical to diagonal to horizontal lines. Yukio Ishihara et al, [3] proposed a method of corrected the distortion of the formed objects in concave mirrors by pre warping the original one. They used many samples of light sources and put it as groups of dots. Then for each of the light sources, the corresponding conjugate points were calculated with ray tracing in a computer model of the concave mirror. This is not only a matter of the number of samples but also they selected equations to use it to model the mapping. So that the observer seen them not distorted and finally they confirm their approach by both computer simulation and a simple experiment. Deepthi Boyanapally [4] corrected the barrel distortion in the projector by first estimating the optical center of the projector and then determining the radial distortion coefficients. The optical center of the projector was calculated assuming that the projector optical center usually lies at the bottom of the projector screen. After the optical center is determined, they manually vary the radial distortion parameter and recalculate the image coordinates of the projected grid. Then used these optical center and radial distortion coefficients in the Multi fingerprint scanner program to radial correct the multi frequency patterns projected on to the 3D object in the scanning process. Usami et al. [5] derived and demonstrated new method for de warping images depicted in convex mirrors in artwork and for estimating the threedimensional shapes of the mirrors themselves, they assumed 
merely that the mirror was radially symmetric and that there were straight lines (or collinear points) in the actual source scene, they express the mirror's shape as a mathematical series and pose the image de-warping task as that of estimating the coefficients in the series expansion. They compute the mirror shape by solving a deferential equation that based on the estimated de-warping function. Rahul Swaminathan et al. [6] introduced a method for finding the distortion parameters that would map the image curves to straight lines by selecting a set of points along those curves. To recover the image parameters a minimized objective function was designed to account for noise in the selected image points. Faisal Bukhari et al. [7] proposed a method for automatic radial distortion estimation based on the plumb-line approach. Their method is based on Fitzgibbon's division model, robust estimation of circular arcs, and robust estimation of distortion parameters. The authors performed an empirical study of the method on synthetic images then finally they provided qualitative results on a wide variety of real image. Gergely Vassy and Tamás Perlaki [8] introduced a semi-automatic tool that is capable to handle the distorted images that fits the need of postproduction studios facilities. The authors had chosen a process based on straightening curved lines that are supposed to be straight to calibrate the lens distortion parameters of actual cameras.

All the previous works try to correct a special type of deformation while this work introduces a general algorithm to correct all the deformations that generated by a fixed and known properties system so this paper will use a convex mirror as fixed and known properties deformation system. The main idea is extracting a deformation map then used it to correct deformed images that captured from a convex mirror. The main elements of this map are the ratios of the rows and of the columns dimensions of a deformed image with respect to their dimensions in the original non deformed image. A small prototype system with convex mirror and digital image has been implemented to capture the required scenes.

This paper is organized as follows; the next section describes the deformation media. Section 3 introduces the proposed prototype system, while the proposed algorithms are described in section 4. The result of testing the capability of the deformation-map in correcting other images is introduced in section 5, and section 6 presents the conclusion of this work

\section{DEFORMATION MEDIA}

There are several reasons behind image deformations, one of the main is the median used to capture the scene. Such median causes the image to lose some information or cause the movement of the image points locally which leads to error in the positions and the values of image points. Some examples are; the effects of the atmosphere on satellite images, the transmission data between two channels, medical equipment such as microscope devices, digital cameras, lenses, scanner devices, concave mirrors, etc. A convex mirror is a curved mirror in which the reflective surface bulges toward the light source, and it reflect light outwards as shown in Fig. 1.

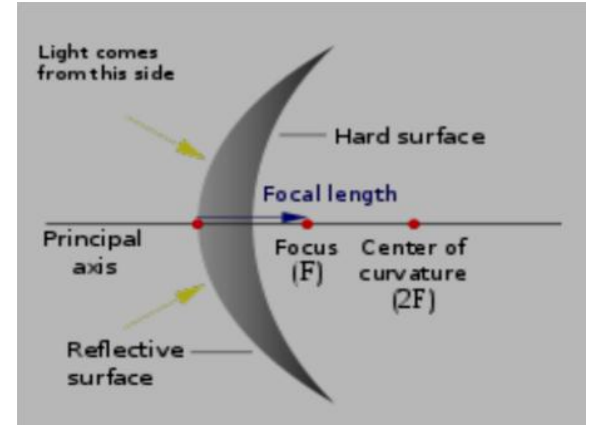

Fig. 1: convex mirror

Such mirrors always form smaller, upright, deformed, and virtual image, since the focus $(\mathrm{F})$ and the center of curvature (C) are both imaginary points "inside" the mirror, which cannot be reached. As a result, images formed by these mirrors cannot be projected on a screen, since the image is inside the mirror. Also they provide a wider field of view as they are curved outwards and allow large area to be viewed. Such mirrors are used in different positions like grocery stores, hospitals, malls and factories. Convex mirror can widen the view of traffic that can be seen in rearview or side view mirrors of automobiles. Distances and sizes seen in convex mirror are not realistic [6]. As explain in Fig. 2 the image reflected from convex mirror appears deformed and virtual so it is necessary to correct that deformation by foundation of image processing and extracted the map of deformation.

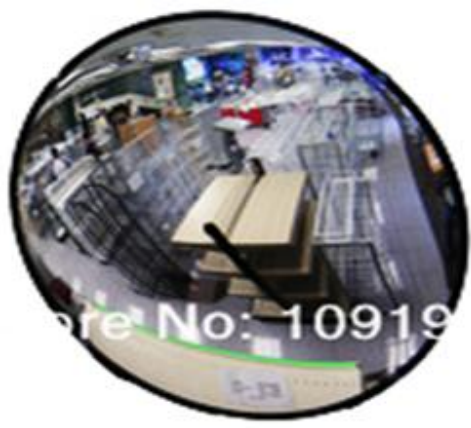

Fig. 2: Indoor convex security mirror

\section{PROPOSED PROTOTYPE SYSTEM}

This work is dealing with the problem of correcting the deformed images reflected by convex security mirrors. This type of mirrors commonly used for surveillance or safety purposes. Convex mirrors (Fig. 2) can be used indoors in supermarkets, hotels, stores, offices, etc. also they can be used outdoors in roads, traffics, as well as in portable inspections.

In order to treat and interpret the images reflected from such mirrors, a digital camera must be used to capture the required scene. In general, the convex mirrors are mounted on a wall or ceiling, thus the camera must be well-positioned in front of the mirror.

In this work, a small prototype system has been constructed. This system is composed of a convex mirror of radius $7.5 \mathrm{~cm}$ and a digital camera of type Sony (14.1MB resolution, and $26 \mathrm{~mm}$ wide-angle lens). It is assumed that the distance 
between the mirror and the image of the target is about $27 \mathrm{~cm}$. This is the optimal viewing distance between the target and the used mirror to maintain a good visibility. This distance can be changed to be more or less, but the larger the distance is the smaller the reflected image, and vice versa. The proposed arrangement for the used system is shown in Fig. 3.

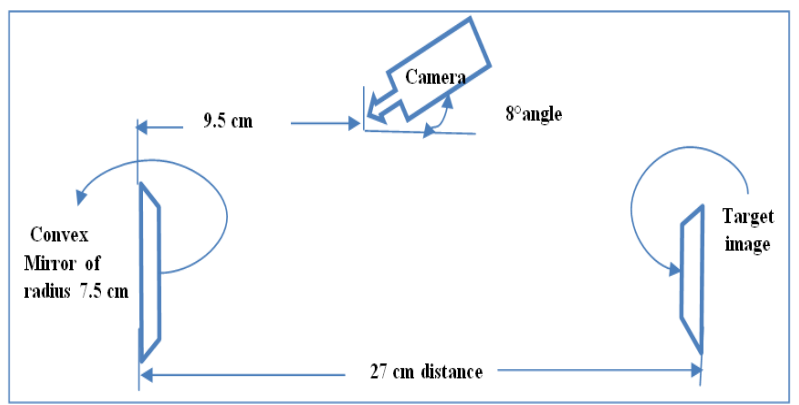

Fig. 3: Block diagram of the proposed system

\section{PROPOSED ALGORITHMS}

The captured images reflected from the convex mirror are deformed and need to be corrected. This work proposed two phases correction process. In the first phase, an algorithm is designed to extract a deformation-map based on some ratios between the deformed image of an object and its original corrected version. The second phase is partitioned to two parts; the first part is to correct the deformed image using the deformation-map, while the second part is to test the capability of generalizing this map to be used in correcting other deformed images.

\subsection{Extracted deformation-map (EDM)}

In this phase an algorithm is designed to extract the deformation-map. This map depends mainly on the compression ratios between two images, the source image of an object and its deformed image (reflected from the convex mirror and captured by the camera of the proposed prototype system). The EDM algorithm computes the compression ratios for each row and each column between the two images to extract the map. To explain the details of this algorithm; let A be the source image of an object, and B is its deformed captured image. The EDM algorithm starts by computing the distances between the first and the last informatics' pixels for each row lies between the first and last column of image A (the term "informatics pixel" used here is refer to a pixel with nonzero values), and then computes the distances for each column lies between the first and last row of the same image. As a result, two distance vectors are obtained; column distance vector $\mathrm{D}_{\mathrm{CA}}$ and row distance vector $\mathrm{D}_{\mathrm{RA}}$. Then, for the deformed image $\mathrm{B}$, the algorithm computes the informatics' column and row distance vectors $D_{C B}, D_{R B}$ respectively. An example illustrates such distances is shown in Fig. 4. Since image B is the compressed version of image A (due to the effects of the convex mirror), there is a high probability that there are some rows and/or some columns in image B have no any informatics pixel. This will lead to generate a distance vector with some zero values components. To put out the effects of any zero value, EDM algorithm treats these components by making their values equal the value of the nearest nonzero component in their corresponding vector. (a)

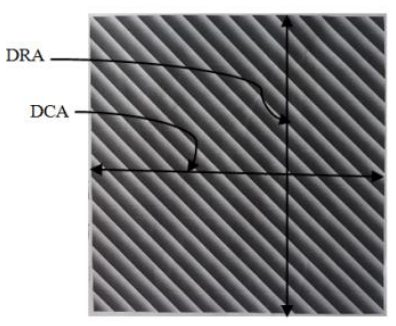

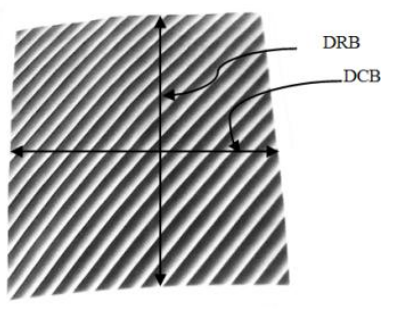

(b)
Fig. 4: (a) Original image A, (b) Deformed image B

The second step in EDM algorithm is to calculate the compression distance ratios for each column and each row in image A with respect to image $\mathrm{B}$. The column distance ratios vector $R C$ can be obtained by dividing each component in vector $\mathrm{D}_{\mathrm{CB}}$ by the corresponding element of vector $\mathrm{D}_{\mathrm{CA}}$. Accordingly, the row ratios vector $R R$ can be obtained by dividing each component in the row distances vector $D_{R B}$ of image $\mathrm{B}$ by the corresponding component in the row distances vector $D_{R A}$ of image $A$. These two ratios vectors are expressed by equation (1) and (2) respectively.

$$
\begin{gathered}
R C_{m}=\frac{D_{C A_{m}}}{D_{C B_{m}}} \\
R R_{n}=\frac{D_{R A_{n}}}{D_{R B_{n}}}
\end{gathered}
$$

for $m=1,2, \ldots, M$, and $\mathrm{n}=1,2, \ldots, N$, where $M$ is the total number of rows between the first and last columns in image A, and $N$ is the total number of columns between the first and last rows in image $\mathrm{A}$.

The row ratio vector $\mathrm{RR}$ and the column ratio vector $\mathrm{RC}$ are the two components of the deformation map. A virtual image representation to this map can be prepared by constructing a matrix based on these two ratios vectors. Each element in this matrix can be computed according to eq. (3) by determining the radius value $R_{m, n}$ which represents the polar coordinate for the corresponding Cartesian element in each of the two ratios vectors.

$$
R_{m, n}=\sqrt{\left(R C_{m}^{2}+R R_{n}^{2}\right)}
$$

The matrix $R$ can be displayed as a virtual image to the deformation-map as shown in Fig. 5. It is clear that the edges zones in this map are less darkness than the center zone. This is due to the differences between the compression ratios, in which the compression ratio in the edges zones is higher than that of the center zone, and that is really what can be seen when using convex mirror. Since the map is displayed as a grayscale image with pixel values between [0-255], we can conclude that, the most darkness pixel can have a value about one, which means the minimum compression ratio, while the less darkness pixel can have a value about 255 , which means the maximum compression ratio. The extracted map is the main tool used in correcting the deformed images in the proposed work. 


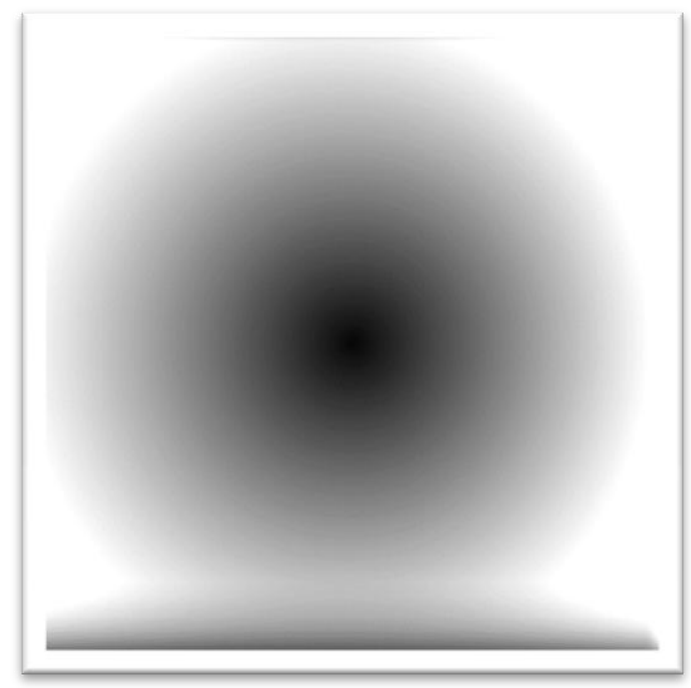

Fig. 5: Deformation map

\subsection{Correction of deformed images}

Correction of deformed images is the second phase of the proposed algorithms. This phase is partitioned to two parts, as mentioned above. The first part is dealing with the correction of the deformed image based on the use of the compression ratios vectors of the deformation-map obtained by the first phase algorithm. While the second part is to test the capability of this map to be used in correcting other images, in other word to check the capability of considering the deformation-map as a general pattern in correcting other deformed image captured by the proposed system, which is explained in the next section.

The idea of the correction algorithm is based on stretching each row and each column in image $B$ to a length based on the corresponding component's value of the compression ratios vectors of the deformation-map. The new length is given by

$$
\begin{aligned}
& L C_{n}(\text { new })=L C_{n}(\text { old }) * R R_{n} \\
& L R_{m}(\text { new })=L R_{m}(\text { old }) * R C_{m}
\end{aligned}
$$

Where $L C_{n}($ new $), L C_{n}($ old $)$ are the new and the current length of column $n$ respectively in image $\mathrm{B}$, and $L R_{m}$ (new), $L R_{m}($ old $)$ are the new and the current length of row $m$ respectively.

The new stretched lengths obtained by eq. (4) are not equal to each other, so as the case with the results obtained from eq. (5). The proposed algorithm fits this problem by using padded arrays with zero values to substitute the differences in lengths. The length of each pad array is calculated as follows,

$$
\begin{aligned}
& P L C_{n}=k * L C_{n}(\text { old })-L C_{n}(\text { new }) \\
& P L R_{m}=l * L R_{m}(\text { old })-L R_{m}(\text { new })
\end{aligned}
$$

Where $P L C_{n}$ and $P L R_{m}$ are the pad length of column $n$ and row $m$ respectively, and, $k$ and $l$ are integer numbers, with

$$
k \geq \frac{1}{\max \left(R R_{n}\right)}, l \geq \frac{1}{\max \left(R C_{m}\right)}
$$

The padding arrays are added to the end of each row and each column with their corresponding length. As a result, the size of the corrected image is enlarged to more than the original size. At this stage, the algorithm cropped the image from the side contains the padding array to the designated size. The output pixels values are covered using bilinear interpolation by calculating the weighted average of pixels in the nearest 2 $\mathrm{x} 2$ neighborhood.

As example on the above mentioned operations is shown in Fig. 6, in which the original image (a) is captured as a deformed image as in (b), adding padding array to the end of the columns is shown in (c), while the image in (d) is the cropped image. The obtained result shown in (d) is not convincing, and still there is a lot of deformity in the cropped image. This is due to stretching the rows and columns in one direction, adding the padded arrays in that direction and cropping the image from that side. Basing on the above mentioned ideas (stretching-padding-cropping), the algorithm is modified to improve its output result.

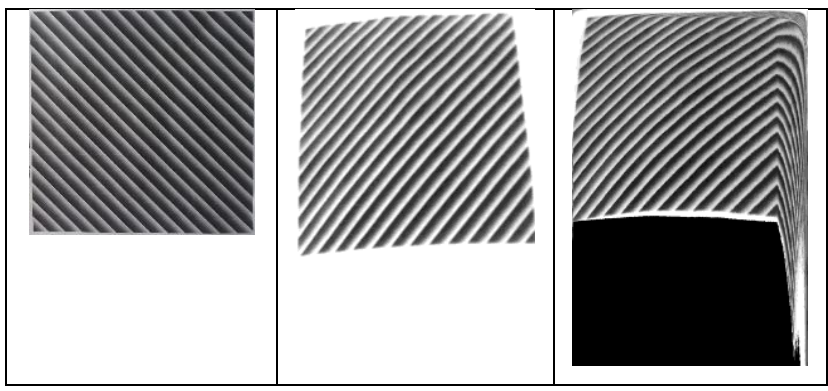

(a)

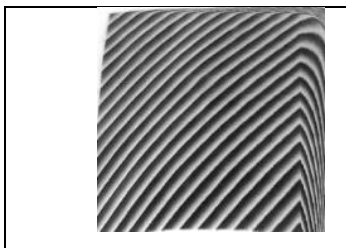

(d) (b)

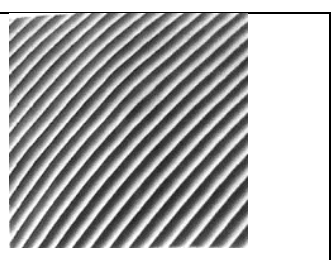

(e)
Fig. 6 : (a) Original image (b) Deformed image (c) Image with padding array (d) Image after cropping (e) Corrected image.

The modification in the algorithm adopted the stretching process of each of the rows and columns to be in both sides rather than in one side. Accordingly, the padding is going to be in both sides. As a result, cropping the treated image will be in both sides of rows and both sides of columns. Stretching the rows and the columns in both sides is completed in two steps, each step stretch in one direction. Thus, the stretching length obtained by eqs. (4) and (5) needs to be modified. The new stretched length for each direction can be obtained by multiply the current length of each column or row by the square root of the corresponding compression ratio,

$$
\begin{aligned}
& L C_{n}(\text { new })=L C_{n}(\text { old }) * \sqrt{R R_{n}} \\
& L R_{m}(\text { new })=L R_{m}(\text { old }) * \sqrt{R C_{m}}
\end{aligned}
$$

The process of stretching in any direction is accomplished by adding pad arrays at the end side, interpolating the values of the pixels, and cropping the image to remove the extra size.

The correction of the deformed image using the modified algorithm brings out good results, as shown in (e) of Fig. 6. 


\section{DEFORMATION-MAP TESTING}

One of the main objectives in this work is to build a general model that can be used in correcting the deformed images. This section tests the capability of the deformation-map to be used as a general correction model for the proposed system.

The first test uses the extracted deformation-map in correcting a 16x16 binary checkerboard image shown in Fig. 7, in which (a) is the original image, (b) is it's deformed captured image, while (c) is the correction result of the deformed image.

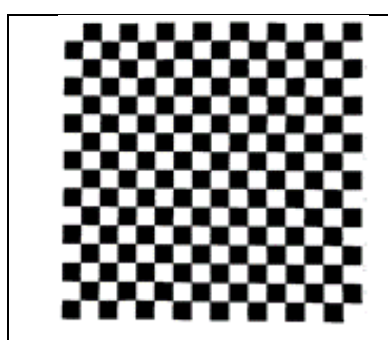

(a)

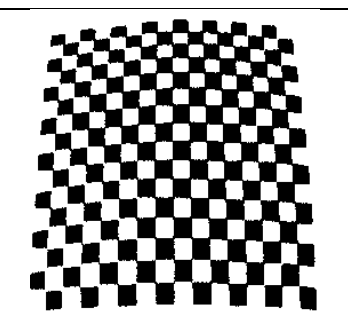

(b)

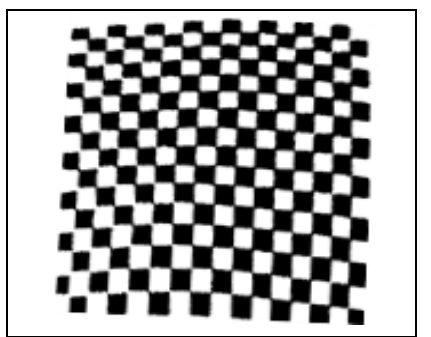

(c)

Fig. 7: Testing Extracted deformation-map on binary checkerboard image (a) Original image (b) Deformed image (c) Corrected image

The second test has been made on correcting the grayscale image of Fig. 8 using the extracted deformation-map. As in Fig. 7, the original, deformed, and corrected images are shown in (a), (b), and (c) respectively.

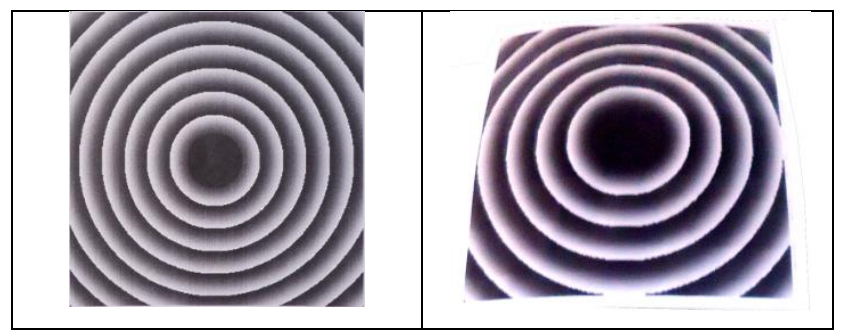

(a)

(b)

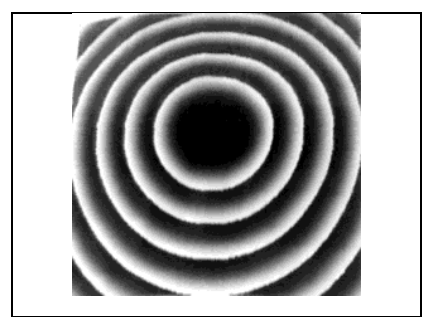

(c)

Fig. 8: Testing Extracted deformation-map on grayscale image (a) Original image (b) Deformed image (c) Corrected image.
The other test is to check the ability of the map to correct color images. RGB or true-color images composed of 3-D arrays. These arrays are the red, green and blue (RGB) image channel component respectively. Each array can be considered as distinct 2-D plane [10]. Thus, in order to correct a color image using the deformation-map, the first step is to separate the red, green and blue planes of the image from each other. Then each plane is corrected by applying the proposed algorithm. The corrected planes are joined to each other to recompose the overall corrected image. Fig. 9 shows the results of correcting two RGB image examples.

It can be concluded that, although the deformation-map is extracted based on image other than those used in this test, but the output of using it as a pattern correction model showed good satisfactory results.

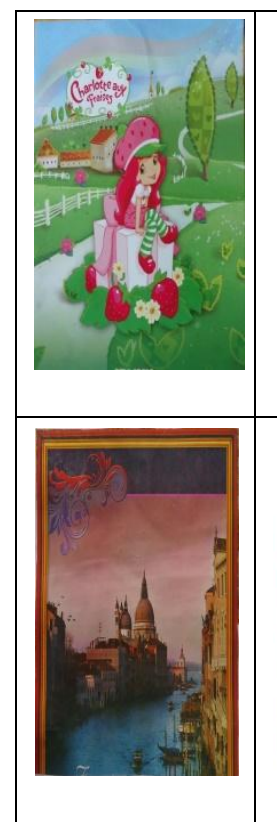

(a)

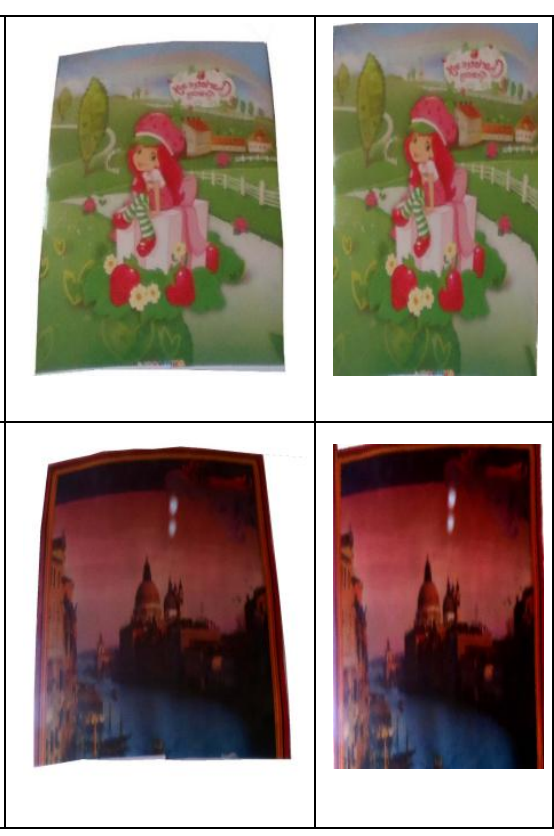

(b)

(c)
Fig. 9: Testing the extracted deformation-map on RGB colored images (a) Original image (b) Deformed image (c) Corrected image

\section{DISCUSSION AND CONCLUSION}

Several image application techniques deal with accurate positional data. Image acquisition devices may have distortion in their lenses which cause displacement in image points. This approach addresses the problem of correcting images deformed through its reflection from convex mirror. The main contribution in this work is concentrated on extracting a deformation map that can be used as a general model to correct the deformation in such images. Two phases correction algorithm have been designed to extract the deformation-map and to correct the deformation in images using this map. The extracted map composed of two main vectors. The components of these vectors are the calculated length ratios of the informatics pixel in each row and column in the deformed image with respect to its corresponding values in the original image. The main operations comprised in the proposed correction algorithm are stretching, adding pad arrays, and cropping. The stretching takes place in both sides of each row and column of the deformed image. The stretched length depends on the corresponding component 
value of the deformation-map. Since the stretched length differ from row to row and from column to column and to make the length of all the rows and all the columns equally sized, a padding arrays are added to the end of the stretched side. The last operation in the proposed algorithm is removing the extra size from each direction. The values of the stretched pixels are obtained using bilinear interpolation by calculating the weighted average of the nearest $2 \times 2$ neighborhood pixels. In order to check the capability of the deformationmap to be used as a general correcting pattern, several deformed images of different types (binary, gray, and colored) have been tested, the obtained corrected output showed very satisfactory results.

\section{REFERENCES}

[1] Ardeshir Goshtasby, "Correction of image deformation from lens distortion using Bezier patches", Department of computer science, university of Kentucky, Lexington, Kentucky 40506-0027, 1989.

[2] Fakhrul Yusoff, Rahmita Rahmat, M. Nasir Sulaiman, Mohamed H Shaharom, Hariyati S. A. Majid, "Establishing the Straightness of a Line for Radial Distortion Correction Through Conic Fitting" IJCSNS International Journal of Computer Science and Network Security, VOL.9 No.5, May 2009.

[3] Yukio Ishihara, Makio Ishihara," Correcting Distorted Objects Formed in a Concave Mirror" UIST'08,October 19-22, 2008, Monterey, California, USA ACM 978-1$59593-975-3 / 08 / 1$.
[4] Deepthi Boyanapally, "Merging of fingerprint scans obtained from multiple cameras in 3D fingerprint scanner system", University of Kentucky Master's Theses, 2008.

[5] Usami, David G. Stork, Jun Fujiki, Hideitsu Hino, Shotaro Akaho, and Noboru Murataa, "Improved methods for de warping images in convex mirrors in fine art: Applications to van Eyck and Parmigianino", Computer vision and image analysis of art II, 2011.

[6] Rahul Swaminathan and Shree K. Nayar, "Non-Metric Calibration of Wide-Angle Lenses and Polycameras", Computer Vision and Pattern Recognition - CVPR, vol. 2, pp. 2413-419 Vol. 2, 1999

[7] Faisal Bukhari and Matthew N. Dailey, "Automatic Radial Distortion Estimation from a Single Image", Journal of Mathematical Imaging and Vision, Vol. 45, Issue 1, pp 31-45, Jan. 2013

[8] Gergely Vassy and Tamás Perlaki, "Applying and removing lens distortion in post production", Proceedings of the Second Hungarian Conference on Computer Graphics and Geometry, Budapest, Hungary, 2003.

[9] Charles McLaughlin, "Integrated Physics and Chemistry-Texas", McGraw-Hill, 2011, ISBN: 0078231418 / 9780078231414.

[10] Chris Solomon, Toby Breckon, "Fundamentals of Digital Image Processing”, 2011 by John Wiley \& Sons, Ltd. 